\title{
Companies vie for a cut of the gene-editing market
}

Researchers hoping to write the next chapter of biomedicine know that their progress will hinge largely on their ability to edit genes-cutting out unwanted DNA and manipulating the sequences they want to keep. Acolytes of gene-editing technology have recently been encouraged by early data about Sangamo BioSciences' new zinc finger nuclease-based therapies. In March, the California biotech reported preliminary phase 1 trial results showing that its HIV treatment, which uses editing to turn off the gene for the receptor on the cell membrane that the virus exploits, was safe and effective at improving people's $\mathrm{T}$ cell counts. Two months later, Sangamo scientists presented additional evidence at the American Society of Gene \& Cell Therapy meeting in Seattle demonstrating in mouse and cell models the technology's promise in a number of other diseases, including hemophilia, so-called 'bubble boy disease' and a form of acquired blindness.

Sangamo's success has fed a growing enthusiasm for genome engineering technologies. And now, a handful of biotechs with competing technologies and business models are vying to be the go-to company for gene-editing applications. But not all technologies are created equal, and there's debate within the research community about which technique is best for which purpose. "It's a bit like 'which one will win out-PCs or Macs?", says Bert Vogelstein, a cancer researcher at the Johns Hopkins University School of Medicine in Baltimore. "The one people will choose is the one that's available, easiest, cheapest and the one they have experience with."

Sangamo's zinc finger nucleases (ZFNs), which remain the best-known gene-editing technique for adult cells, can latch onto DNA at specific points and cleave unwanted parts out, in some cases repairing a disease-causing gene. In 2007, Sangamo licensed sales of the technology for research purposes to the St. Louis-based company Sigma-Aldrich, which now charges $\$ 25,000$ for new constructs. Philip Gregory, Sangamo's chief scientific officer, admits that's a considerable price to pay. But he notes that even at that price point, demand is outpacing supply. "We've been asking Sigma to reduce the prices so that we could get the technology out there faster," he says. "But they claim to be overwhelmed with orders, anyway."

Across the Atlantic, Horizon Discovery, a Cambridge, UK startup founded in 2007, is advancing its own gene-editing platform using another pitch. Although not as efficient as ZFNs, Horizon's approach is more flexible: it can add genes as easily as it can delete them,

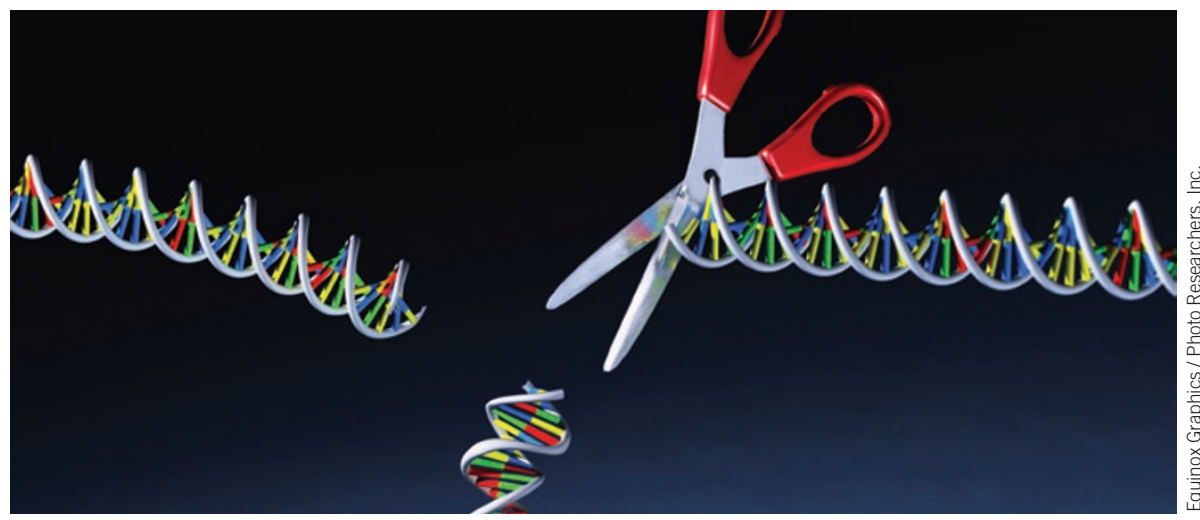

Scissor lift: Companies hope for big profits from gene-editing technologies.

and the technology is easier to track.

Horizon's technology relies on harmless adeno-associated viruses (AAVs) that can deliver a desired gene sequence to a defined location in the human chromosomes via homologous recombination, and it has started to garner some attention from big pharma. In June, for instance, the company announced the completion of an 18-month pilot program developing human cell lines for the Swiss pharma giant Novartis. But, in general, AAVs have fallen under the radar, partly because the technology hasn't had large corporate backing, according to David Russell, a geneticist at University of Washington School of Medicine in Seattle and the technique's inventor.

To spread the word, Horizon began assembling a consortium of academic and nonprofit institutions to exchange free AAV constructs and technical support for a licensing agreement on any cell lines the institutions produced using its approach. (Ordinarily, Horizon charges corporate clients $\$ 50,000$ for a custom cell line or $\$ 20,000$ for a stock line.)

\section{Technology par excellence}

So far this year, six institutions have signed up as so-called Centers of Excellence, including the Fox Chase Cancer Center in Philadelphia and the Translational Genomics Research Institute in Phoenix, among others. The company plans to enlist 44 more institutions by the end of the year, including, notably, the US National Institutes of Health in Bethesda, Maryland.

Teaming up with Horizon "was a winner all the way around," says Fox Chase's chief scientific officer Jonathan Chernoff. "We needed a more realistic model of what we encounter in a clinical study." And AAVs, he argues, allows his team to create and study the same mutations in cell cultures that physicians find in patients.

Horizon's head of research and development,
Rob Howes, hopes the Centers of Excellence will encourage scientists to share technological improvements at the same time as they spread the platform into more research areas. "AAV has been used extensively in cancer research," he notes, "but there are a lot of other disease areas to expand into, like cardiovascular and neuroscience."

Meanwhile, in February, Paris-based Cellectis shook up the market by signing a licensing agreement with the Universities of Iowa and Minnesota for the sale of transcription activator-like effector nucleases, or TALENs. The enzymes work much like zinc fingers to home in on and snip specific DNA sequences. But unlike ZFNs, which bind in groups of three base pairs, TALENs bind individual nucleotides.

"Zinc fingers have whetted everyone's appetite for genome modification," says Dan Voytas, a geneticist at the University of Minnesota-Twin Cities and one of the original TALEN designers. "Everyone is really excited." Cellectis has already begun offering custom TALENs to researchers starting at $\$ 5,000$.

There are a number of ways to judge the merits of each technique, but there's no consensus about which is quickest or easiest-and each has its drawbacks. AAVs, for example, only take hold in around one in a million cells, which is fine for creating a disease model in the lab, but not for gene therapy. TALENs and ZFNs, for their part, are more efficient, but they have been criticized for cutting DNA at off-target sites that can be hard to trace. "There are problems with all of them," Vogelstein says.

Thus, it remains unclear which technology will emerge as the field's gold standard. "My hunch as a bench scientist?" asks Voytas. "I don't know."

Daniel Grushkin 